\title{
La croissance de l'emploi en Espagne de 1995 à 2005
}

El crecimiento del empleo en España de 1995 a 2005

Analysis of characteristics of employment growth in Spain between 1995 and 2005

Inmaculada Cebrian

\section{OpenEdition}

\section{Journals}

Édition électronique

URL : http://journals.openedition.org/travailemploi/4204

DOI : 10.4000/travailemploi.4204

ISSN : 1775-416X

\section{Éditeur}

DARES - Ministère du Travail

\section{Édition imprimée}

Date de publication : 15 septembre 2008

Pagination : 29-44

ISSN : 0224-4365

\section{Référence électronique}

Inmaculada Cebrian, «La croissance de l'emploi en Espagne de 1995 à 2005 », Travail et Emploi [En ligne], 115 I juillet-septembre 2008, mis en ligne le 18 février 2011, consulté le 09 mai 2019. URL http://journals.openedition.org/travailemploi/4204; DOI : 10.4000/travailemploi.4204 


\title{
La croissance de l'emploi en Espagne de 1995 à 2005
}

\author{
Inmaculada Cebrián (*)
}

L'Espagne a connu depuis la crise économique qu'elle a traversée au début des années 1990 un taux de croissance moyen ininterrompu de 3,4\% par an, soit près de deux points de plus que les pays de l'Union européenne à quinze. Le niveau de l'emploi fut favorisé par cette longue phase d'expansion, qui s'accompagna d'une forte croissance de la population active, due non seulement à l'augmentation du taux d'activité des Espagnols mais aussi à l'arrivée en masse d'immigrants (voir les articles de Toharia et Cachón dans ce numéro). Ce processus permit de réduire le taux de chômage et les différentiels du taux de population active occupée avec l'Union européenne. Il a été conduit dans la recherche d'une véritable convergence, même si les niveaux de productivité n'ont pas augmenté.

Le modèle de croissance espagnol souffre cependant de certains problèmes liés au fait que les principes sur lesquels il s'appuie sont par excellence la consommation privée et l'expansion du secteur de la construction, alors que la balance commerciale reste faible. De plus, malgré une certaine modération des salaires, le coût du travail a connu une plus forte augmentation en Espagne que dans les pays de l'Union européenne à quinze et de la zone euro, tandis que la productivité augmentait moins et que le différentiel du coût unitaire du travail se maintenait. Par ailleurs, la croissance de l'emploi s'est concentrée sur quelques secteurs et métiers en relation directe avec l'importance croissante que prenaient les secteurs de la construction et des services aux personnes, tous deux caractérisés par des niveaux de productivité assez faibles.

Autre fait important: une part considérable de l'emploi créé est associée à des contrats à caractère

(*) Universitad de Alcalá, Departemento de Fundamentos de Economiá e Historia Economica; immaculada.cebrian@uah.es temporaire et à des emplois à bas salaires (voir plus loin Miguelez et Prieto pour plus de détails). De plus, l'Espagne compte encore une part importante de travailleurs manuels peu qualifiés, et c'est ce type d'emplois qui attire une main-d'œuvre immigrée manuelle, ce qui génère des problèmes de ségrégation professionnelle. Ces caractéristiques sont à l'origine d'emplois instables à faible productivité et à bas salaires.

Cet article analyse l'influence de ces facteurs sur la création d'emplois et donne un aperçu de l'évolution de la structure de l'emploi par secteur de production et par métiers qui pourrait conditionner la croissance de l'économie espagnole. Enfin, il cherche à déterminer si le volume et le type d'emplois créés suffiront à garantir les niveaux de croissance et de bien-être de la population.

L'article est construit en deux temps. Dans un premier temps, il décrit certaines caractéristiques et spécificités de l'évolution des principaux indicateurs macro-économiques liés à l'évolution récente de l'emploi en Espagne: la production et la productivité du travail, les rémunérations et les coûts unitaires du travail. La seconde partie de l'article est consacrée plus spécifiquement à l'explication détaillée de l'évolution et de la structure de l'emploi. Il analyse pour cela l'évolution des secteurs d'activité, des professions, des contrats et du temps de travail entre 1995 et 2006. Enfin, il conclut sur les conséquences futures des problèmes potentiels détectés sur le marché du travail espagnol. Ces analyses et la comparaison avec les quatre plus grands pays européens (France, Allemagne, Italie et RoyaumeUni) et avec les pays de l'Union européenne à quinze comme groupe de référence sur la période 1995-2005, ont été réalisées à partir des données de l'Institut national de la statistique espagnol (INE) et d'Eurostat sur les comptes nationaux et les enquêtes sur les forces de travail. 


\section{Évolution de l'emploi et des autres grandeurs macro-économiques}

L'article de Luis Toharia décrit les caractéristiques de l'activité, de l'emploi et du chômage pour les dernières décennies et les périodes d'expansion et de récession qui se sont succédées. Nous nous concentrerons ici sur l'évolution de l'emploi pendant la phase d'expansion qui débute en 1995, en analysant d'autres variables macro-économiques: production, productivité du travail, rémunération et coûts unitaires du travail(1). Nous verrons que d'importantes différences dans l'évolution de ces agrégats séparent l'Espagne des quatre principaux pays européens (France, Allemagne, Italie et Royaume-Uni).

\section{Production, emploi et productivité du travail}

Le graphique 1 donne les taux de croissance de la production, de l'emploi et de la productivité entre 1978 et 2005 et décrit la relation entre ces variables au cours des différentes phases de l'économie espagnole. Ces chiffres montrent que la production et de l'emploi ont, surtout depuis les années 1980, la même évolution respective dans les périodes de crise que dans les périodes d'expansion, et les différences entre les deux séries indiquent les conséquences à tirer en termes de productivité. Après la crise la plus profonde qu'ait connue l'Espagne au début des années 1990, plus courte mais plus intense que celle des années 1980, son économie a traversé une période de croissance extraordinaire, qui se reflète principalement dans l'augmentation remarquable de l'emploi, en progression très nette.

On distingue clairement quatre périodes depuis 1978: la première correspond à une crise de l'emploi qui a duré jusqu'au milieu des années 1980; la seconde à une période de reprise, de 1985 à 1990; la troisième, jusqu'en 1994, se caractérise par la crise économique la plus profonde; et la dernière, qui débute en 1995, est une période de longue croissance en termes de production et d'emploi. Cette dernière période de dix ans est particulièrement intéressante dans la mesure où la croissance de l'emploi et de la production sont presque identiques, alors que la productivité est très faible. On voit qu'emploi et production sont positivement corrélées durant la croissance économique des périodes d'expansion; de fait, la croissance de l'emploi a été un facteur décisif pour la durabilité de la dernière phase d'expansion(2). Ceci a permis la convergence avec les pays européens les plus développés, le différentiel de productivité persistant néanmoins.

(1) Depuis 1995, les statistiques sont relativement uniformes. (2) La création d'emplois, accompagnée de conditions financières favorables, permet à la consommation privée et au secteur de la construction d'être les moteurs des dépenses intérieures.
Selon la théorie macro-économique générale, la croissance de la productivité est la seule source d'amélioration de l'économie, une fois que la croissance de l'emploi a atteint son niveau maximum. La productivité dépend de la relation entre capital physique, emploi et changements technologiques. Sur le long terme, une croissance économique stable n'est basée que sur une amélioration de la productivité fondée sur des avancées technologiques. Sur le court terme, la productivité affiche un comportement procyclique, c'est-à-dire que sa tendance suit l'orientation de l'activité économique. Cependant, l'une des caractéristiques les plus étonnantes du modèle économique espagnol est que la croissance de la production et de l'emploi se sont accompagnées d'un mouvement contraire de la productivité apparente du travail. Ce comportement anticyclique est tout à fait particulier et tout à fait contraire à ce que l'on observe dans d'autres économies. Le graphique 1 permet de voir comment la productivité avait tendance à augmenter à un rythme relativement rapide lorsque l'emploi baissait, fluctuant d'une période à l'autre. Tel a été le cas lors de la crise de 1975-1985, quand l'économie espagnole a affiché des gains considérables de productivité au moment où de nombreux emplois étaient supprimés et où la croissance de l'emploi était négative. Le contraire s'est produit lors de la période de croissance du milieu des années 1980, le taux de croissance de la productivité étant beaucoup plus faible, surtout en 1990. Lorsque la crise a de nouveau frappé au début des années 1990 , l'économie a retrouvé son modèle précédent. Mais après 1994, quand la production et l'emploi connaissaient une croissance à un taux positif et régulier, la productivité a atteint son plus haut niveau en 1995 (5\%). Depuis, elle a notablement baissé et est actuellement très faible (environ $1 \%$ ). Des raisons structurelles pourraient expliquer cette décélération. Une première hypothèse pourrait être qu'il s'est produit une réaffectation sectorielle des emplois avec un déplacement vers les secteurs à plus faible productivité, ainsi qu'une incapacité à exploiter de nouvelles technologies et de nouveaux investissements, la part des emplois peu qualifiés restant au contraire très importante. Le taux très élevé des contrats à caractère temporaire, comme l'expliquent TOHARIA (2002) et les experts espagnols (COMISIÓN DE EXPERTOS PARA EL DIÁLOGUO SOCIAL, 2005), peut également constituer un facteur significatif. Le fait que la plupart des emplois créés se soient concentrés sur des professions et des secteurs d'activité à faible productivité, alors que les contrats temporaires abondaient, sont des facteurs qui entravent l'amélioration de la productivité.

Cependant, la chute de la croissance de la productivité ne s'est pas constatée uniquement en Espagne, mais aussi dans d'autres pays européens tels que l'Allemagne, l'Italie et la France depuis le milieu des années 1990 (JiMENo et al. 2006). Néanmoins, le tableau 1 présente les taux de croissance annuels du PIB, de l'emploi et de la productivité de ces pays 


\section{Graphique 1 : Évolution des taux de croissance de la production, de l'emploi et de la productivité en Espagne (1978-2005)}

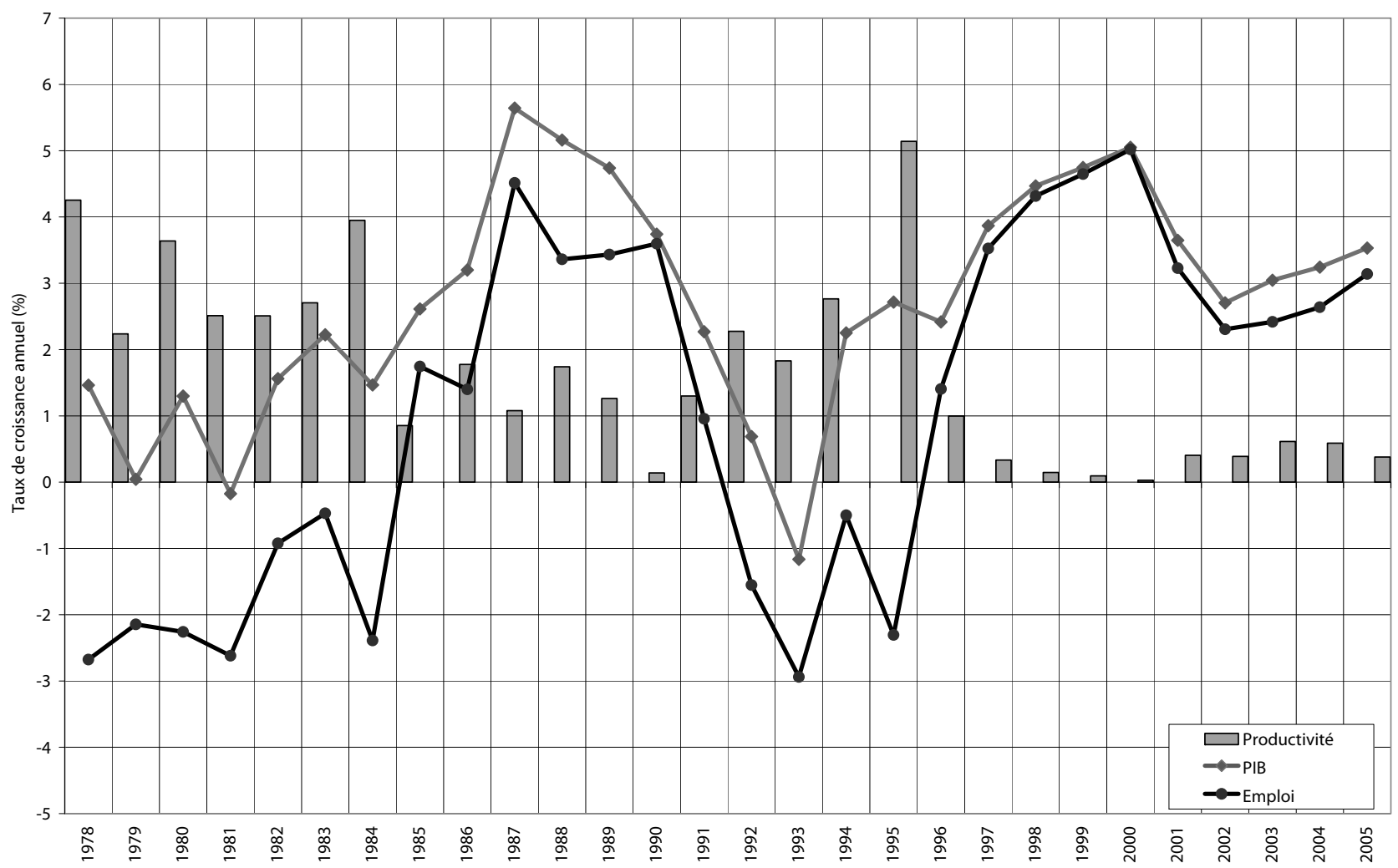

Source: Institut national des statistiques espagnol (INE), Comptes nationaux espagnols, année de base: 2000 (*).

(*) La mesure de la production utilisée est le produit intérieur brut (PIB). L'emploi est mesuré en équivalents temps plein. La productivité est la production par travailleur en équivalents temps plein et se réfère également à la productivité apparente du travail.

en 1996 et en 2006 et nous pouvons voir que l'Espagne a connu la hausse la plus notable du PIB et de l'emploi, mais aussi une chute de la productivité durant cette décennie.

Tableau 1: Taux de croissance du PIB réel, de l'emploi et de la productivité en 1996 et 2005 (3)

\begin{tabular}{|c|c|c|c|c|c|c|}
\hline & \multicolumn{3}{|c|}{1996} & \multicolumn{3}{|c|}{2005} \\
\hline & $\begin{array}{l}\bar{D} \\
\stackrel{\circlearrowright}{0} \\
\stackrel{0}{0}\end{array}$ & 它 & 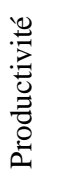 & 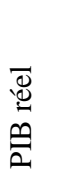 & 㝙 & $\begin{array}{l}\stackrel{0}{0} \\
.00 \\
0 \\
0 \\
0 \\
0\end{array}$ \\
\hline $\begin{array}{l}\text { Union européenne } \\
\text { à quinze }\end{array}$ & 1,7 & 0,6 & 1,1 & 1,6 & 0,9 & 0,7 \\
\hline Espagne & 2,4 & 1,7 & 0,7 & 3,6 & 4,1 & $-0,5$ \\
\hline Allemagne & 1 & $-0,3$ & 1,3 & 0,8 & -01 & 0,9 \\
\hline France & 1,1 & 0,4 & 0,7 & 1,7 & 0,4 & 1,3 \\
\hline Italie & 0,7 & 0,6 & 0,1 & 0,1 & 0,3 & $-0,2$ \\
\hline Royaume-Uni & 2,8 & 1 & 1,8 & 1,8 & 0,9 & 0,9 \\
\hline
\end{tabular}

Source: Eurostat.

(3) Les principaux taux de croissance des agrégats sont disponibles depuis 1996 pour les pays de l'Union européenne à 15 .
Au milieu des années 1990, l'économie espagnole est entrée dans une phase de croissance modérée accompagnée d'une période de croissance intensive de l'emploi, mais d'un taux de productivité très bas. Comme nous l'étudierons plus loin, la croissance économique pourrait être entravée par le type d'emplois créés.

\section{Part du travail dans le revenu national et évolution des coûts unitaires du travail}

La question de l'évolution de la masse salariale et de sa part dans le revenu national est importante pour comprendre l'évolution positive de la production et de l'emploi. Rappelons que la production (le produit intérieur brut (PIB)) est la somme des revenus de l'économie sur une période donnée.

Le tableau 2 présente la répartition de la valeur ajoutée par types de revenus en Espagne entre 1995 et 2005 , et indique la part de chacun dans les performances économiques globales. Nous constatons 
que, depuis 1995, la masse salariale(4) a augmenté à plusieurs reprises, de 48,8\% du PIB total en 1995 à $49,6 \%$ en 2000 , et connu une baisse en 2005 , où elle atteint seulement $47 \%$. Cette forte diminution de la part de la masse salariale dans le PIB peut être liée aux changements positifs constatés aussi bien dans le volume de l'excédent brut d'exploitation et le revenu mixte que dans les impôts indirects; ces derniers ont évolué dans le sens contraire de la masse salariale; quand l'une diminue, les autres augmentent. Cependant, la part des impôts indirects, qui représentent le reste, augmentent dans le revenu national depuis 1995 . Cette évolution peut être source de problèmes, pour les salariés comme pour les employeurs, et affecter la productivité. Le taux de croissance annuelle du revenu du travail se situe aujourd'hui autour de $6,1 \%$, celui du revenu du capital monte à $8,6 \%$, et celui des impôts indirects à $12 \%$, ce qui est très préoccupant. La pression fiscale plus élevée en termes de PIB est directement liée aux fortes augmentations des prélèvements qui ont eu lieu suite à l'augmentation de la consommation et à l'évolution négative des prix du pétrole. Le problème principal réside dans le fait que ces revenus fiscaux ont augmenté régulièrement plus que la croissance économique.

Le tableau 3 compare la part de la rémunération du travail en Espagne et dans les principaux pays européens, en relation avec la valeur ajoutée brute en prix courants, c'est-à-dire le PIB hors taxes. Nous constatons que l'économie espagnole présente une part de rémunération du travail et un pourcentage de salariés plus faibles que les autres pays, à l'exception de l'Italie. Nous nous intéressons aux implications en termes de répartition du revenu.

Sur le plan de la demande, l'évolution des coûts unitaires du travail (CUT) est plus importante que l'évolution générale des revenus du travail. La croissance du coût unitaire du travail met en rapport la rémunération et la productivité et montre comment et dans quelle mesure la rémunération du travail est liée à la productivité du travail. C'est la relation entre le salaire que perçoit le travailleur et la quantité qu'il produit.

D'après la signification du concept de coût unitaire du travail (CUT), exprimé en termes de ratio de masse salariale par unité de travail et de productivité du travail, un pays peut améliorer sa compétitivité, soit en diminuant le coût du travail par salarié, soit en augmentant les performances de la productivité. Une économie peut donc avoir recours à différentes stratégies pour améliorer la compétitivité, par exemple en modérant l'augmentation des salaires afin de réduire les coûts, en

(4) En contraste avec la mesure précédente de la productivité, les mesures des revenus indiquées dans le tableau 1 sont exprimées en prix courants et non ajustés pour le prix relatif. augmentant la productivité pour accroître la production, ou en combinant les deux de manière appropriée. Le tableau 4 présente les taux de croissance annuelle du coût unitaire de travail réel et nominal de différents pays de l'Union européenne. Malgré une baisse récente de son CUT réel et nominal, l'Espagne possède l'un des niveaux les plus élevés de CUT. La chute des CUT pourrait être due à une réduction plus importante de la proportion de la masse salariale dans le revenu national espagnol, qui n'a pas été compensée par une augmentation de la part des salariés.

En résumé, nous pouvons affirmer qu'il existe des preuves évidentes de la réduction progressive de la compétitivité de l'économie espagnole. La productivité en termes réels a été très faible ces dernières années, et, malgré le fait qu'elle coïncide avec l'évolution négative des coûts unitaires du travail (CUT), les CUT espagnols sont plus élevés que dans le reste de l'Union européenne à quinze. Les variables montrent que la productivité n'augmente pas en Espagne et que la baisse des CUT ne suffit pas à améliorer la compétitivité.

Nous étudierons en détail dans la partie suivante la structure de l'emploi en tenant compte non seulement de l'évolution de l'emploi mais aussi de la part croissante de certains secteurs d'activités et métiers, ainsi que les types de relations du travail entre employeurs et salariés selon les types de contrats utilisés et les régimes de temps de travail.

\section{Structure de l'emploi et changements récents sur le marché du travail espagnol}

Comme nous l'avons vu, une question clé pour comprendre ce qui s'est passé dans l'économie espagnole ces dix dernières années réside dans le fait d'avoir créé des emplois dans des secteurs et métiers spécifiques, ce qui pourrait avoir pour effet d'entraver la croissance de la productivité et d'augmenter les disparités entre la productivité des différents secteurs économiques.

\section{Structure sectorielle de l'emploi}

Deux indicateurs permettent de mesurer les changements structurels de la production et de l'emploi dans les différents secteurs d'activité. Le premier se base sur le calcul de la valeur ajoutée des secteurs économiques par rapport à la totalité de la valeur ajoutée de l'économie espagnole. Nous pouvons observer dans le tableau 5 des différences intéressantes entre la production et sa répartition par secteurs d'activité et les taux de croissance, en Espagne, dans Union européenne à quinze et dans deux pays européens significatifs, la France et l'Italie. 
Tableau 2: Composition du PIB par types de revenus (en \%)

\begin{tabular}{|l|c|c|c|c|}
\hline & En \% & \multicolumn{3}{|c|}{ Taux de croissance sur dix ans en \% } \\
\hline Composition du PIB & $\mathbf{1 9 9 5}$ & $\mathbf{2 0 0 0}$ & $\mathbf{2 0 0 5}$ & $\mathbf{1 9 9 5 - 2 0 0 5}$ \\
\hline Masse salariale & 48,8 & 49,6 & 47,0 & 94,9 \\
\hline $\begin{array}{l}\text { Excédent brut d'exploitation } \\
\text { et revenues mixtes? }\end{array}$ & 42,6 & 40,5 & 42,1 & 100,3 \\
\hline Impôts nets indirects & 8,6 & 9,9 & 10,9 & 156,7 \\
\hline Total & 100,0 & 100,0 & 100,0 & \\
\hline
\end{tabular}

Source: Institut national de la statistique espagnol (INE), Comptes nationaux espagnols, 1995-2005.

Tableau 3 : Part de la masse salariale sur la valeur ajoutée brute (VAB)* en prix courants, proportion des salariés sur la totalité des emplois et taux de croissance des coûts unitaires du travail (CUT)

\begin{tabular}{|l|c|c|c|c|c|c|}
\hline & \multicolumn{2}{|c|}{$\begin{array}{c}\text { \% de la masse salariale dans la } \\
\text { valeur ajoutée brute }\end{array}$} & \multicolumn{2}{|c|}{$\begin{array}{c}\text { Part des salariés dans } \\
\text { l'emploi }\end{array}$} & $\begin{array}{c}\text { Taux de croissance } \\
\text { annuelle réel du coût du } \\
\text { travail }\end{array}$ \\
\hline & $\mathbf{1 9 9 5}$ & $\mathbf{2 0 0 5}$ & $\mathbf{1 9 9 5}$ & $\mathbf{2 0 0 5}$ & $\mathbf{1 9 9 5}$ & $\mathbf{2 0 0 5}$ \\
\hline Union européenne à quinze & 56,3 & 55,6 & 84,1 & 85,5 & & -04 \\
\hline Espagne & 53,0 & 52,5 & 90,0 & 88,9 & -42 & -16 \\
\hline Allemagne & 59,7 & 55,9 & 81,2 & 85,8 & 0,0 & -17 \\
\hline France & 57,9 & 58,3 & 89,1 & 91,0 & -04 & 0 \\
\hline Italie & 45,7 & 45,5 & 73,1 & 76,0 & -33 & 0,8 \\
\hline Royaume-Uni & 58,2 & 59,8 & 84,4 & 86,4 & -12 & 1,5 \\
\hline
\end{tabular}

* La VAB est le PIB hors taxes.

Source: Eurostat et OCDE.

Tableau 4: Taux de croissance annuelle du coût unitaire de travail réel et nominal (1996 et 2005)

\begin{tabular}{|l|c|c|c|c|}
\hline & \multicolumn{2}{|c|}{ CUT* } & \multicolumn{2}{c|}{ CUTR** } \\
\hline & $\mathbf{1 9 9 6}$ & $\mathbf{2 0 0 5}$ & $\mathbf{1 9 9 6}$ & $\mathbf{2 0 0 5}$ \\
\hline Union européenne à quinze *** & 1,83 & 1,05 & -08 & -04 \\
\hline Espagne & 3,03 & 2,54 & 0,1 & -16 \\
\hline Allemagne & 0,22 & -095 & -05 & -17 \\
\hline France & 1,47 & 1,66 & -03 & 0 \\
\hline Italie & 5,70 & 2,88 & 0,3 & 0,8 \\
\hline Royaume-Uni & 1,19 & 3,39 & -24 & 1,5 \\
\hline
\end{tabular}

Source: Eurostat et OCDE.

Remarques :

*CUT $(\mathrm{OCDE})=$ coût unitaire du travail : coût total nominal par employé/productivité du travail réel.

**CUTR (Eurostat): coût unitaire de travail réel: salaire par employé en prix courants divisé par le PIB en prix courants par l'emploi total. *** Zone euro au lieu de l'Union européenne à quinze pour l'analyse du CUT. 


\section{Encadré 1 \\ Mesure de la productivité à partir des comptes nationaux espagnols}

La productivité du travail s'obtient en divisant le produit Intérieur Brut (PIB) (réel) en euros constants par le nombre de travailleurs en équivalent temps pleins employés dans l'économie sur une année de base. Cette information est fournie par les Comptes nationaux espagnols (CNE), dont le système se base sur le système européen de comptabilité de 1995 (SEC-95) et dont les estimations sont ajustées sur les mêmes principes de cohérence et d'équilibre budgétaire. II faut rappeler que le PIB est la valeur des biens et services produits par le travail et le capital présents sur le territoire espagnol, et non celui que fournissent les résidents espagnols, comme c'est le cas pour le produit national brut (PNB).

D'un autre côté, il faut prendre en compte certains problèmes empiriques lorsque nous nous intéressons à l'estimation de la productivité. II nous faut un indicateur de l'emploi cohérent lié à la mesure du niveau de production. C'est pourquoi nous avons utilisé les données issues des CNE plutôt que l'indicateur de l'emploi habituellement utilisé fourni par l'enquête sur la population active (EPA). Certaines différences peuvent être observées entre les deux sources d'informations, comme nous allons l'expliquer.

Les Comptes nationaux espagnols évaluent le niveau de l'emploi et fournissent des informations sur le nombre total de personnes employées, le nombre d'emplois et le volume d'emploi en équivalents temps pleins.

Le concept d'emploi est le même pour les deux sources d'informations, CNE et EPA, à une différence significative près: I'EPA prend en compte des travailleurs résidant depuis au moins un an en Espagne dans un foyer non collectif, produisant des biens pour l'Espagne à l'intérieur ou à l'extérieur du pays. Une autre différence provient de la définition de l'emploi salarié car les travailleurs en coopérative sont inclus dans cette catégorie dans les CNE suivant les recommandations du SEC-95, tandis que l'EPA les classe dans les professions indépendantes.

Lorsque les CNE calculent le nombre d'emplois, ils prennent en compte tous les emplois. Par conséquent, un travailleur qui a plusieurs emplois est comptabilisé autant de fois que le nombre d'emplois qu'il occupe. Ce n'est pas le cas dans l'EPA. Par ailleurs, le concept d'emploi équivalent temps plein fourni par les CNE prend en compte la quantité totale d'heures effectuées. Dans ce cas, deux travailleurs à temps partiel employés à mi-temps sont considérés comme un seul travailleur à temps complet.

En 2005, les CNE ont changé l'année de base de 1995 à 2000, et ont adapté le niveau de l'emploi aux nouvelles données disponibles sur la population et la démographie à la suite du recensement de 2001. Les augmentations importantes concernant le nombre d'immigrés en Espagne ont rendu caduques les prévisions en matière de mouvements de population et d'évolutions démographiques calculées à partir du recensement de 1991. Ces changements ont donné lieu à une augmentation considérable des niveaux de l'emploi dans les CNE, encore plus importante que dans l'EPA. À la suite de ces modifications, le niveau de l'emploi mesuré par les CNE était plus élevé que celui fourni par l'EPA(1).

II faut donc être particulièrement vigilant dans le calcul des niveaux de productivité puisqu'ils peuvent être affectés de fortes différences selon les sources relatives au niveau de l'emploi qui sont utilisées. En fait, on pourrait estimer les changements en matière de productivité de façon positive ou négative selon la source utilisée pour mesurer l'emploi.

\section{Le coût unitaire du travail}

L'indicateur du coût unitaire du travail met en rapport rémunération et productivité et est employé comme indicateur de la compétitivité des coûts. Il s'agit d'un rapport construit à partir d'un numérateur (salaire par employé) en termes nominaux et d'un dénominateur (production par employé) en termes réels. Il représente le coût actuel du travail par « unité de quantité » produite et montre de quelle façon la rémunération des travailleurs est liée à la productivité. Son taux de croissance indique donc la dynamique de la part du travail dans la valeur de la production créée.

L'équation du coût unitaire de travail peut s'écrire comme le rapport entre les coûts de travail nominaux par salarié et la productivité réelle du travail par salarié:

$$
\begin{gathered}
\text { Équation (1): } \\
\frac{\text { CUT = RT (t)/ES (t) }}{\mathrm{Y}(\mathrm{t}) / \mathrm{E}(\mathrm{t})}
\end{gathered}
$$

L'équation (1) indique le coût unitaire du travail (CUT) sur une année de base (t). Le numérateur est composé du rapport entre la rémunération du travail (RT), incluant les contributions des employeurs à la Sécurité sociale, et $E S$, qui représente le nombre d'emplois salariés. Le dénominateur est constitué du rapport entre $Y$, la production à partir du PIB, et $E$, le nombre total d'employés, dont les professions indépendantes.

Nous pouvons réécrire l'équation (1) pour décomposer la différence en coût unitaire de travail sur la

(1) Pour une analyse détaillée des différences entre CNE et EPA, voir PEREz INFANTE (2006) 
production à partir de la part des employés salariés par le total des personnes employées, comme le montre l'équation (2). Mais il est également possible de montrer que la diminution de la part des salaires dans le PIB peut s'expliquer par une évolution combinée de l'augmentation de la part des salariés et d'une diminution du CUT, comme le montre l'équation (3):

$$
\begin{gathered}
\text { Équation (2): } \\
\frac{\operatorname{CUT}(\mathrm{t})=\mathrm{RT}(\mathrm{t})^{*} \mathrm{E}(\mathrm{t})}{\mathrm{Y}(\mathrm{t}) \mathrm{ES}(\mathrm{t})} \\
\text { Équation }(3): \\
\frac{\mathrm{RT}(\mathrm{t})}{\mathrm{Y}(\mathrm{t})}=\mathrm{CUT}^{*} \frac{\mathrm{ES}(\mathrm{t})}{\mathrm{E}(\mathrm{t})}
\end{gathered}
$$

La division des coûts unitaires du travail par la production nominale fait également parfois référence au coût unitaire du travail réel - puisqu'il équivaut à un coût unitaire de travail exprimé en prix constants où le déflateur utilisé est l'indice des prix dérivé du PIB pour l'activité économique.

Théoriquement, une augmentation des coûts du travail peut résulter d'une pression à la hausse des salaires ou d'un ralentissement de la croissance de la productivité. La pression à la hausse des salaires peut être un phénomène externe déclenché par l'appréciation de la monnaie d'un pays. Elle peut également avoir une cause interne, par exemple, à une pénurie de main-d'œuvre. Un ralentissement de la productivité peut être dû à une augmentation dans la part du secteur des services car la productivité des services augmente généralement plus lentement que la productivité du secteur secondaire. Mais une croissance lente de la productivité peut également être due au manque d'avancées technologiques ou à la lenteur des réformes sur les marchés des produits ou du travail. Les causes des changements dans les coûts unitaires du travail ont donc des implications importantes pour les politiques du marché du travail et des produits, les politiques relatives aux technologies et aux innovations ainsi que les politiques de commerce extérieur.

Sur la période étudiée, la valeur ajoutée du secteur agricole est passée de $4,5 \%$ du PIB total à 3,9\% en Espagne, tandis qu'elle reste autour des $3 \%$ dans d'autres pays. En outre, l'emploi dans ce secteur chute dans tous les pays. En ce qui concerne l'Espagne, ce processus est dû à une perte continue de la production et de la force de travail dans ce secteur depuis 1980. Les parts du secteur industriel, de gros et de détail, et du secteur des services non marchands (administration publique et autres services) ont diminué, tandis que la part de la construction et de l'intermédiation financière a remarquablement augmenté dans le PIB total espagnol. Il convient de souligner que cette augmentation significative de la part du secteur de la construction dans le PIB est seulement caractéristique de l'Espagne, tandis que l'intermédiation financière augmente dans tous les pays.

La forte augmentation du nombre de personnes employées dans les deux secteurs, avec des taux respectifs de croissance de $94,2 \%$ et $79,4 \%$, plus élevés que la croissance de la production sur la période de référence, pourrait expliquer la diminution de la productivité. Le graphique 2 compare l'évolution de la croissance de la production et de l'emploi et l'évolution conséquente de la productivité par secteur d'activité en Espagne; il est particulièrement intéressant de comprendre ce qui fait que le secteur de la construction ait atteint un taux de croissance de $12 \%$ à la fin des années 1990. Cela a engendré une très forte diminution de la productivité apparente du travail dans ce secteur, avec des niveaux très bas, sauf en 2002 lors d'un ralentissement.

Le développement de l'emploi dans les secteurs suit des modèles différents. L'analyse de la répartition de l'emploi dans les secteurs d'activité doit être réalisée avec prudence, car cet indicateur peut varier ou non en réponse à l'évolution du taux général de l'emploi, qui dépend toujours du niveau de développement et des changements structurels de l'économie. Néanmoins, dans l'analyse sectorielle, la part des employés dans un secteur peut également varier en fonction de la variation de l'emploi dans d'autres secteurs, puisqu'il s'agit de la composition de l'emploi. Pour comprendre ce qui se passe, il est parfois nécessaire de se reporter à l'évolution des disparités entre différents secteurs économiques. Le graphique 3 présente l'évolution de l'emploi dans les principaux secteurs économiques à partir de 1987, ainsi que l'emploi total(5).

Sur les vingt-cinq dernières années, l'agriculture a perdu près de $50 \%$ de sa force de travail. Bien que le poids de ce secteur dans l'emploi reste plus élevé que le chiffre moyen des autres pays européens, il ne faut pas s'attendre à une diminution importante de l'emploi dans ce secteur (ToHARIA, 2002).

L'emploi dans le secteur industriel présente une tendance cyclique, de façon générale, mais on peut constater une baisse générale par rapport à

(5) Pour une analyse des années précédentes, consulter TOHARIA (2004). 
Tableau 5 :Variation de la production et de l'emploi en pourcentages (1995 et 2005)

\begin{tabular}{|c|c|c|c|c|c|c|c|c|c|c|c|c|}
\hline & \multicolumn{3}{|c|}{ Espagne } & \multicolumn{3}{|c|}{ France } & \multicolumn{3}{|c|}{ Italie } & \multicolumn{3}{|c|}{$\begin{array}{l}\text { Union européenne à } \\
\text { quinze }\end{array}$} \\
\hline & 1995 & 2005 & $\begin{array}{l}\text { Taux de } \\
\text { crois- } \\
\text { sance }\end{array}$ & 1995 & 2005 & $\begin{array}{l}\text { Taux de } \\
\text { crois- } \\
\text { sance }\end{array}$ & 1995 & 2005 & $\begin{array}{c}\text { Taux } \\
\text { de } \\
\text { crois- } \\
\text { sance }\end{array}$ & 1995 & 2005 & $\begin{array}{c}\text { Taux } \\
\text { de } \\
\text { crois- } \\
\text { sance }\end{array}$ \\
\hline \multicolumn{13}{|l|}{ Production } \\
\hline Agriculture et pêche & 4,5 & 3,9 & 23,7 & 3,4 & 3,0 & 10,1 & 3,3 & 3,2 & 7,5 & 2,7 & 24 & 9,3 \\
\hline Industrie & 21,9 & 207 & 33,8 & 19,1 & 19,3 & 25,2 & 25,0 & 21,2 & $-4,6$ & 23,5 & 22,1 & 16,7 \\
\hline Construction & 7,5 & 8,8 & 66,3 & 5,6 & 4,8 & 5,7 & 5,3 & 5,7 & 21,1 & 5,96 & 5,2 & 8,2 \\
\hline Gros et détails $^{(1)}$ & 26,9 & 24,7 & 29,8 & 19,5 & 20,9 & 32,7 & 24,2 & 25,9 & 21,0 & 21,0 & 22,2 & 32,0 \\
\hline Intermédiation financière & 17,9 & 20,5 & 61,3 & 28,1 & 30,1 & 32,8 & 22,3 & 23,8 & 20,4 & 24,4 & 27,0 & 37,5 \\
\hline $\begin{array}{l}\text { Administration publique et } \\
\text { autres services }\end{array}$ & 21,3 & 20,8 & 37,6 & 24,4 & 21,9 & 11,4 & 19,8 & 20,2 & 14,7 & 22,4 & 21,0 & 16,1 \\
\hline \multicolumn{13}{|l|}{ Emploi } \\
\hline Agriculture et pêche & 7,9 & 5,2 & $-6,4$ & 4,6 & 4,3 & $-3,9$ & 6,0 & 4,1 & $-24,0$ & 4,93 & 3,7 & $-15,5$ \\
\hline Industrie & 19,1 & 16,9 & 25,7 & 17,3 & 16,5 & $-2,2$ & 24,1 & 21,0 & $-3,1$ & 20,2 & 16,7 & $-7,5$ \\
\hline Construction & 9,1 & 12,5 & 94,5 & 6,5 & 6,4 & 1,8 & 6,8 & 7,6 & 25,2 & 7,3 & 7,3 & 12,4 \\
\hline Gros et détails ${ }^{(1)}$ & 27,3 & 27,6 & 43,7 & 22,6 & 22,9 & 3,8 & 24,3 & 24,3 & 11,6 & 25,3 & 25,7 & 14,0 \\
\hline Intermédiation financière & 8,5 & 10,8 & 79,4 & 15,2 & 15,9 & 7,4 & 10,4 & 14,4 & 54,0 & 12,7 & 16,0 & 41,1 \\
\hline $\begin{array}{l}\text { Administration publique et } \\
\text { autres services }\end{array}$ & 28,1 & 27,0 & 36,4 & 33,8 & 34,0 & 3,3 & 28,4 & 28,6 & 12,2 & 29,6 & 30,7 & 16,2 \\
\hline
\end{tabular}

Source: Eurostat, Comptes nationaux.

(1) Le secteur gros et détail comprend le commerce de gros et de détail, les hôtels et les restaurants, le transport, le stockage et la communication.

Graphique 2 : Évolution des taux de croissance de la production, de l'emploi et de la productivité par secteur d'activité en Espagne (1996-2005)

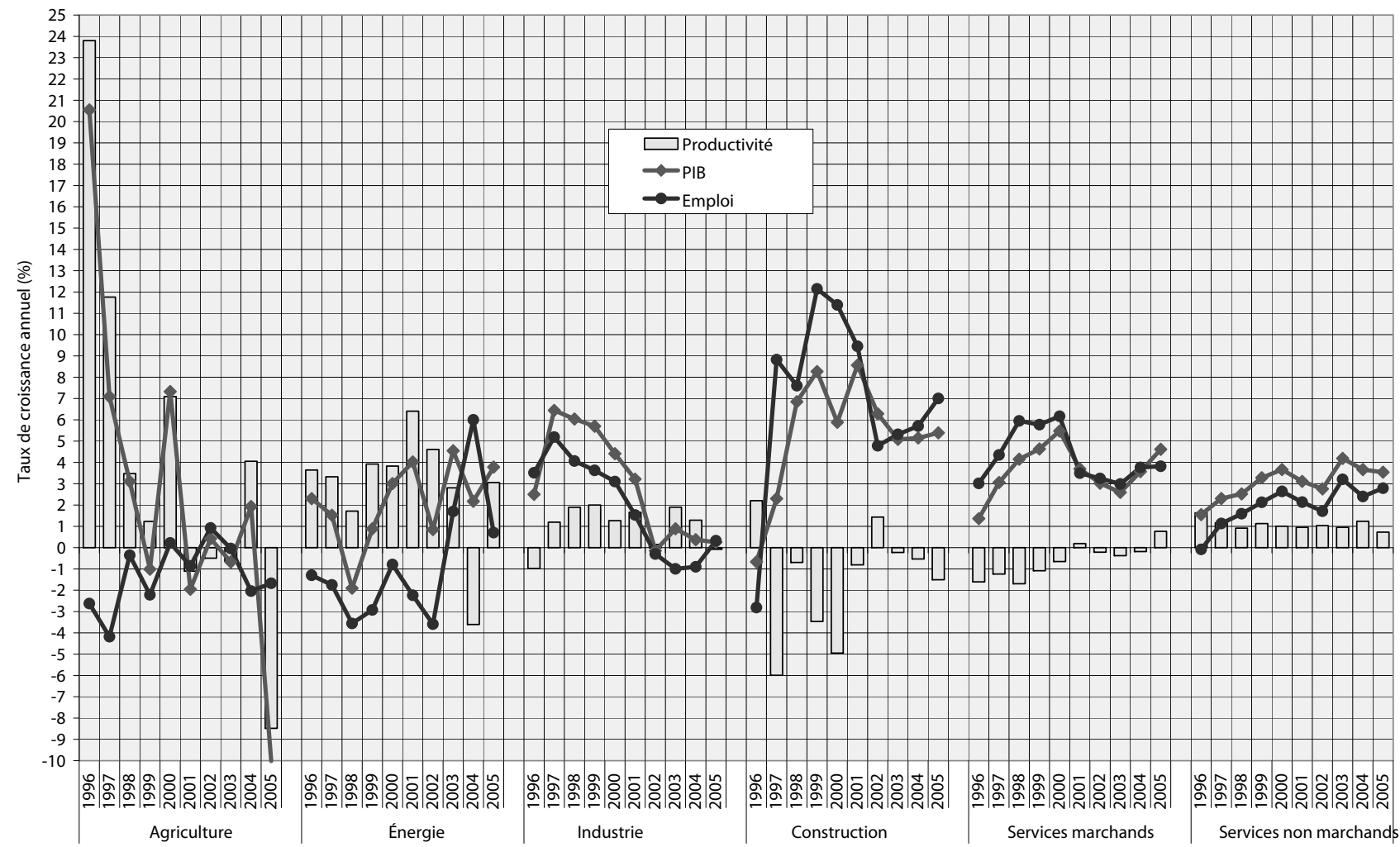

Source: Institut national de la statistique espagnol (INE), Comptes nationaux, année de base 2000. 
Graphique 3 : Évolution de l'emploi sectoriel selon l'enquête sur la population active espagnole (1987-2006) (indice $1987=\mathbf{1 0 0}$ )

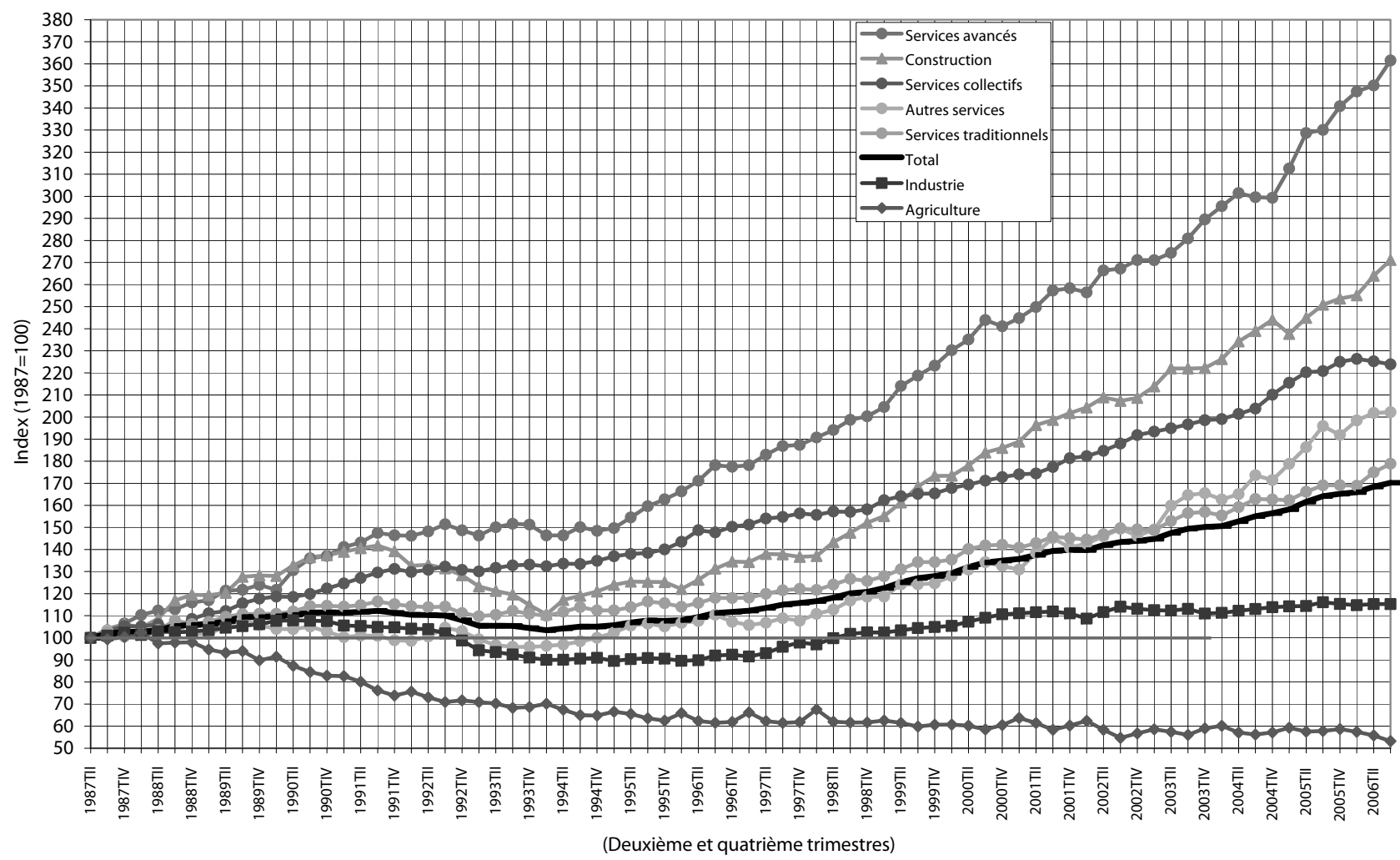

Source: Institut national de la statistique espagnol (INE), enquête sur la population active.

Légende:

- le secteur Agriculture comprend l'agriculture, la chasse et la pêche;

- le secteur industriel comprend les activités de fabrication et l'énergie;

- construction;

- les services traditionnels incluent les services de détail, les hôtels et les restaurants, le transport et la communication, le commerce et les services de communication;

- les services avancés incluent les activités d'entreprise, les services financiers et les agents immobiliers;

- les services collectifs comprennent l'administration publique, la Sécurité sociale et la défense, l'éducation, la santé et les soins vétérinaires;

- les autres services sont les activités sociales, les services personnels et les employés de maison.

l'évolution dans les services. Ainsi, la reprise ces dix dernières années n'a pas été suffisante pour compenser sa forte baisse précédente et les pertes subies lors de la crise des années 1990.

$\mathrm{Au}$ contraire, le secteur de la construction, qui a toujours affiché une tendance nettement cyclique, connaît depuis la dernière crise une reprise durable. À cette époque, et notamment en 1993, ce secteur a connu une fois de plus des pertes considérables au niveau de l'emploi, mais a su rapidement recréer de nouveaux emplois à partir de 1994. Le chiffre a aujourd'hui plus que doublé et a atteint un niveau jamais égalé pour toute la période étudiée (plus élevé d'environ $130 \%$ que celui enregistré en 1995). Sur la totalité de la production et de l'emploi, il est passé respectivement de 7 à $11 \%$ et de 9 à $13 \%$.

L'emploi dans le secteur des services traditionnels maintient la même tendance que la croissance générale de l'emploi tout au long de la période. Ce secteur est directement lié au tourisme, qui est resté assez stable; le secteur conserve sa part de $28 \%$ sur la totalité de l'emploi.

Les services collectifs, comme ceux relatifs à l'administration publique, la santé et l'éducation, ont connu une véritable explosion, néanmoins plus faible que dans le secteur de la construction. Lors de la crise du début des années 1990, ce secteur résistait à la suppression d'emplois et a su recréer davantage d'emplois pendant la reprise, augmentant au même rythme que l'emploi global, sauf entre 2000 et 2003 où il a connu une légère baisse du niveau de changement. L'augmentation nette de l'emploi était clairement plus faible que dans le secteur de la construction, avec moins de $50 \%$ d'augmentation entre 1995 et 2006.

Le modèle développé par les services avancés, service qui semble le plus dynamique, est particulièrement intéressant. Il compte trois fois plus de travailleurs en 2006 qu'en 1987, ce qui signifie qu'il a connu une plus forte augmentation que le secteur de la construction. Il représente ainsi une 
augmentation absolue de 1,7 million d'emplois ou 1,2 dans les dix dernières années. Cette évolution double la part de ce secteur dans l'emploi total, qui était en 1987 de 5,8\% et atteint plus de $11,8 \%$ en 2006, d'après l'enquête sur les forces de travail. Cependant, si nous comparons l'évolution du secteur de la construction et de celui des services avancés depuis le milieu des années 1990, nous constatons une évolution similaire. Ceci s'explique par le fait que les services avancés comprennent des activités directement liées au secteur de la construction, telles que les opérations financières et les activités des agents immobiliers.

L'emploi dans les autres services comme l'assistance aux personnes et les employés de maison, affiche une tendance semblable à celle de l'emploi total jusqu'en 2001, date où il a commencé à augmenter et où les chiffres ont fini par doubler. Ce fait s'explique par l'arrivée de migrants et la demande croissante de services aux personnes et d'employés de maison depuis 1996. Ce secteur représente $8 \%$ de l'emploi total en 2006.

Au total, l'Espagne est donc dotée d'une structure économique spécifique puisque l'agriculture y reste plus élevée que dans les autres pays de l'Union européenne et que le secteur de la construction et des services y ont une plus forte influence sur la structure de l'emploi que dans l'Union européenne à quinze.

Pour résumer, l'emploi total et sectoriel a connu des changements considérables ces dernières décennies, notamment ces dix dernières années, et l'emploi a augmenté de manière continue à un rythme positif bien que des différences importantes persistent entre les secteurs. À la fin du siècle dernier, l'économie espagnole était déjà totalement une économie de services, depuis que le secteur tertiaire représente plus de $60 \%$ de l'emploi total. Par contraste, la reprise qui a débuté en 1994 s'est principalement concentrée sur les secteurs de la construction et des services avancés, et dans une moindre mesure sur les services traditionnels et l'industrie. L'agriculture continue de baisser, moins rapidement cependant ces dernières années. L'augmentation du nombre d'emplois créés permet une convergence avec l'Union européenne, bien qu' un nouveau problème persiste, dû à l'importance significative du secteur de la construction et aux problèmes conséquents de volatilité.

\section{Structure des emplois et changements récents des professions en Espagne}

En réponse aux développements récents du niveau de l'emploi, la structure des emplois aurait pu être modifiée, à la suite de la mise en place de nouvelles technologies, mais aussi du fait des changements dans la structure des secteurs d'activités. Comme nous venons de l'expliquer, la croissance du secteur de la construction et des autres activités liées à ce secteur est impressionnante en Espagne. L'apparition de plusieurs nouveaux métiers, ainsi que le maintien d'autres plus traditionnels peuvent en être la conséquence. Lorsque de nouvelles méthodes de production et d'organisation nécessitent de nouveaux types de compétences, comme cela peut être le cas dans le secteur des services avancés, de nouveaux métiers peuvent surgir; cependant, le secteur de la construction se caractérise par un besoin en qualifications peu élevées et par une structure d'emploi basique. Nous nous concentrerons donc maintenant sur l'évolution des différentes catégories de métiers afin d'observer si cette nouvelle situation a affecté la composition des professions et de l'emploi.

Les éléments qui suivent sur la composition et l'évolution de l'emploi dans différents types de métiers entre 1995 et 2006, sont tirés des données de l'enquête sur la population active.

Tableau 6 : Indicateurs de la structure de l'emploi en Espagne en pourcentages (1995 et 2006)

\begin{tabular}{|c|c|c|c|}
\hline & \multicolumn{2}{|c|}{ Emploi total } & \multirow{2}{*}{$\begin{array}{c}\text { Taux de } \\
\text { changement }\end{array}$} \\
\hline & 1995 & 2006 & \\
\hline \multicolumn{4}{|l|}{ Part } \\
\hline Non manuel & 50,3 & 56,5 & 80,6 \\
\hline Manuel & 49,7 & 43,5 & 41,1 \\
\hline \multicolumn{4}{|l|}{ Part } \\
\hline $\begin{array}{l}\text { Compétences } \\
\text { peu élevées }\end{array}$ & 38,3 & 40,5 & 70,4 \\
\hline $\begin{array}{l}\text { Compétences } \\
\text { élevées }\end{array}$ & 61,7 & 59,5 & 55,1 \\
\hline
\end{tabular}

Source: Institut national de la statistique espagnol (INE), enquête sur la population active.

Légende:

Non manuel: directeurs: «législateurs, hauts fonctionnaires et directeurs», "professions libérales», «techniciens et professionnels associés», «forces armées», «employés de bureau», "personnel de service, ouvriers et vendeurs».

Manuel: pêcheurs et agriculteurs qualifiés, artisans et employés de l'artisanat, opérateurs, monteurs et conducteurs des moyens de transport, ouvriers non spécialisés.

Compétences élevées: législateurs, hauts fonctionnaires et directeurs, professions libérales, techniciens et professionnels associés, forces armées, pêcheurs et agriculteurs qualifiés, artisans et employés de l'artisanat, opérateurs, monteurs et conducteurs des moyens de transport.

Compétences peu élevées: employés de bureau, personnel de service et vendeurs, ouvriers non spécialisés.

$\mathrm{Si}$ nous regroupons les professions en deux grandes catégories, les emplois manuels et les emplois non manuels, nous observons qu'une création nette d'emplois a été enregistrée dans les emplois non manuels avec une croissance de $80,6 \%$, pour $41,1 \%$ dans les emplois manuels; les emplois à compétences peu élevées ont connu une hausse de 70,4\%, et les emplois à compétence élevée une croissance de 55,1\%. Par conséquent, nous pouvons en conclure que la création nette d'emploi est principalement liée à l'augmentation d'emplois non manuels ainsi que des métiers néces- 
Tableau 7 : Répartition de l'emploi par professions en pourcentages (1995 et 2005)

\begin{tabular}{|l|c|c|c|c|c|c|c|c|c|c|c|c|}
\hline & \multicolumn{3}{|c|}{ Espagne } & \multicolumn{3}{c|}{ France } & \multicolumn{3}{c|}{ Italie } & \multicolumn{3}{c|}{$\begin{array}{c}\text { Union européenne à } \\
\text { quinze }\end{array}$} \\
\hline & $\mathbf{1 9 9 5}$ & $\mathbf{2 0 0 5}$ & $\begin{array}{c}\text { Taux de } \\
\text { croissance }\end{array}$ & $\mathbf{1 9 9 5}$ & $\mathbf{2 0 0 5}$ & $\begin{array}{c}\text { Taux de } \\
\text { croissance }\end{array}$ & $\mathbf{1 9 9 5}$ & $\mathbf{2 0 0 5}$ & $\begin{array}{c}\text { Taux de } \\
\text { croissance }\end{array}$ & $\mathbf{1 9 9 5}$ & $\mathbf{2 0 0 5}$ & $\begin{array}{c}\text { Taux de } \\
\text { croissance }\end{array}$ \\
\hline Directeurs & 8,3 & 7,5 & 58,0 & 7,9 & 8,3 & 14,2 & 3,2 & 8,6 & 15,1 & 8,5 & 8,9 & 20,8 \\
\hline $\begin{array}{l}\text { Professions } \\
\text { libérales }\end{array}$ & 10,2 & 12,5 & 43,2 & 10,4 & 13,2 & 19,9 & 9,4 & 9,6 & 211,5 & 11,7 & 13,8 & 27,1 \\
\hline Techniciens & 7,4 & 11,5 & 92,8 & 16,8 & 18,0 & 45,7 & 14,2 & 21,4 & 17,8 & 13,8 & 17,0 & 41,9 \\
\hline $\begin{array}{l}\text { Employés de } \\
\text { bureau }\end{array}$ & 10,1 & 9,4 & 144,7 & 14,4 & 11,9 & 22,3 & 13,5 & 11,2 & 73,8 & 13,8 & 11,8 & 48,4 \\
\hline $\begin{array}{l}\text { Personnel de } \\
\text { service }\end{array}$ & 14,0 & 15,4 & 47,4 & 11,9 & 12,7 & $-5,3$ & 15,8 & 11,2 & $-4,8$ & 13,1 & 13,8 & 3,7 \\
\hline $\begin{array}{l}\text { Agriculteurs } \\
\text { qualifiés }\end{array}$ & 6,8 & 2,7 & 74,0 & 5,0 & 3,9 & 21,6 & 4,2 & 2,2 & $-18,9$ & 4,0 & 2,8 & 27,3 \\
\hline $\begin{array}{l}\text { Artisans et } \\
\text { employés de } \\
\text { l'artisanat }\end{array}$ & 17,4 & 16,6 & $-37,1$ & 13,9 & 12,1 & $-11,4$ & 20,0 & 16,4 & $-39,1$ & 16,4 & 13,5 & $-15,3$ \\
\hline $\begin{array}{l}\text { Opérateurs } \\
\text { des moyens de } \\
\text { transport }\end{array}$ & 11,1 & 9,3 & 50,8 & 10,8 & 9,0 & $-0,7$ & 9,4 & 8,9 & $-5,4$ & 8,9 & 7,9 & -08 \\
\hline $\begin{array}{l}\text { Ouvriers non } \\
\text { spécialisés }\end{array}$ & 14,4 & 14,7 & 33,4 & 7,5 & 9,6 & $-5,1$ & 9,4 & 9,4 & 9,3 & 8,9 & 9,9 & 6,4 \\
\hline Forces armées & 0,4 & 0,4 & 61,5 & 1,3 & 1,2 & 46,6 & 0,9 & 1,1 & 14,4 & 0,8 & 0,6 & 33,8 \\
\hline
\end{tabular}

Source: Eurostat, enquête sur la population active.

sitant peu de compétences, bien que le nombre de nouveaux métiers à compétences élevées soit plus élevé en chiffres absolus et représente près de $60 \%$ de la totalité des nouveaux emplois en 2006. D'un autre côté, la part des professions nécessitant peu de compétences dans l'emploi total a augmenté ces dix dernières années, puisque le taux est passé de $38,3 \%$ à $40,5 \%$.

Le tableau 7 présente une analyse plus détaillée des différentes catégories de professions et leur répartition dans l'emploi total. Nous comparons également le cas espagnol à celui d'autres pays européens, la France et l'Italie, et de l'Union européenne à quinze.

Comme nous l'avons précédemment souligné, nous pouvons observer qu'une grande partie de la croissance récente de l'emploi en Espagne concerne clairement les métiers non manuels, avec une importance relativement plus élevée des plus qualifiés. Il est particulièrement intéressant de constater l'évolution positive des métiers de niveau moyen tels que les techniciens et professionnels associés, dont la part a presque doublé. Cependant, si l'on compare l'Espagne à d'autres pays européens, des différences significatives font surface. Premièrement, la part des emplois non manuels à compétences élevées (directeurs, professions libérales et techniciens) est toujours plus faible qu'en France, qu'en Italie et que dans l'Union européenne à quinze. La part des techniciens dans ces pays atteint presque $20 \%$. Le chiffre est inférieur de 8 points en Espagne. $\mathrm{Au}$ contraire, les emplois nécessitant des compétences peu élevées ont également connu une croissance importante en Espagne. La part de cette catégorie est légèrement plus élevée en 2006 qu'en 1995. Les emplois manuels exigeant des compétences élevées présentent le taux de croissance le plus bas et leur part reste stable sur la période étudiée. Ce comportement général peut s'expliquer par l'évolution générale de l'emploi en Espagne et la concentration des augmentations dans le secteur de la construction et des services liés à ce secteur. Si l'on compare la situation de l'Espagne à celles de la France et de l'Italie, on constate que dans ces deux pays, les opérateurs de machines et les ouvriers d'usine sont en déclin, ainsi que les ouvriers non spécialisés en France avec un taux de croissance négatif, à côté de leur forte part dans l'emploi total.

De la même manière que dans la partie précédente, nous analyserons maintenant si ces changements sont dus à l'évolution générale de l'emploi ou à un changement de la structure de l'emploi en Espagne. Le graphique 4 présente l'évolution de différents métiers, en prenant 1995 comme année de référence. Cette analyse nous permet d'évaluer l'importance de chaque profession sur dix ans. 
Graphique 4 : Évolution de l'emploi dans les différentes professions, 1995-2006, (indice 1995 = 100)

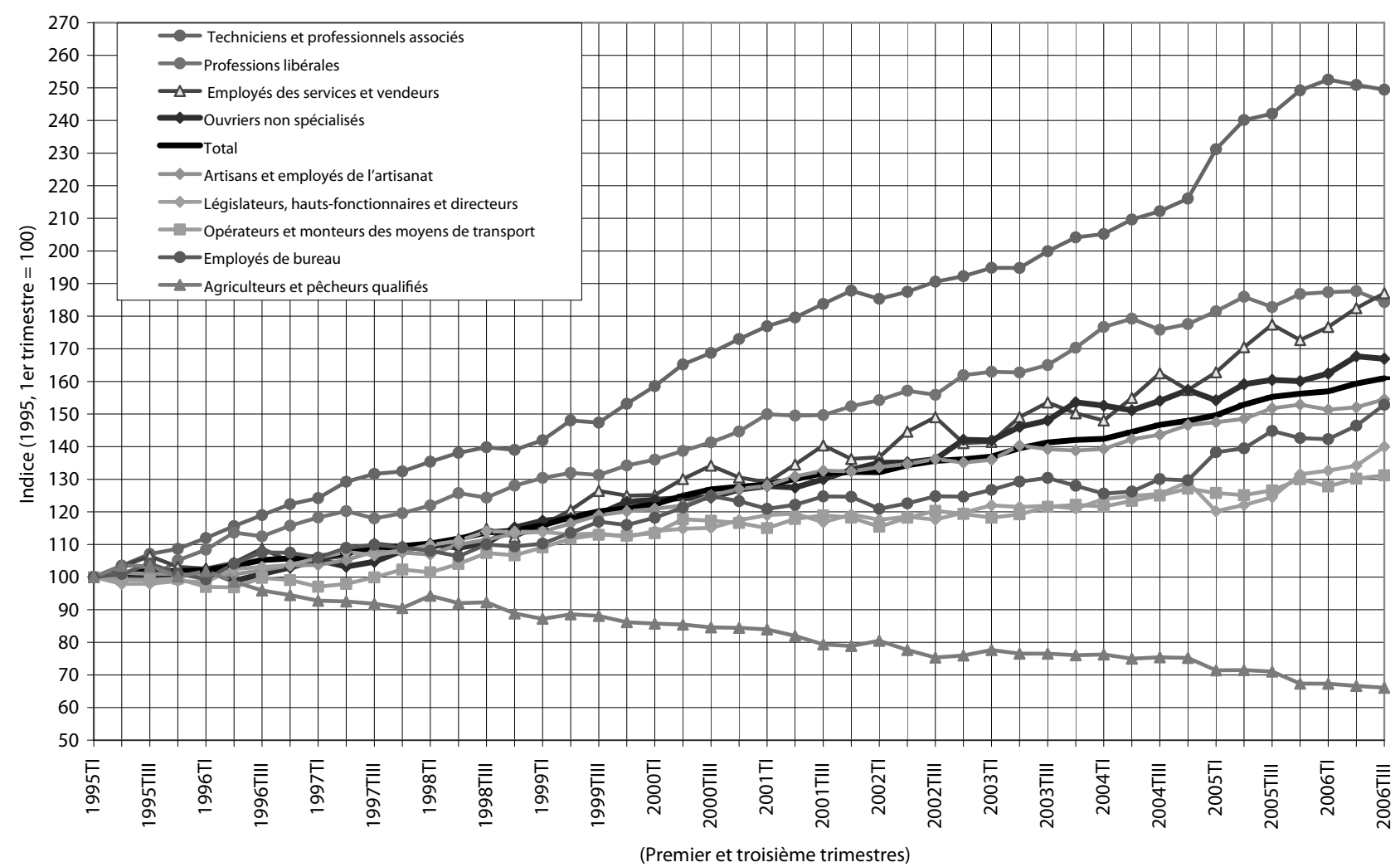

Source: Institut national de la statistique espagnol (INE), enquête sur la population active.

En ce qui concerne l'évolution de la structure des professions sur le marché du travail espagnol, le graphique 4 montre une grande dispersion des taux de croissance des différents métiers de 1995 à 2006. Il faut souligner que certains métiers non manuels à compétences élevées se situent au-dessus de la moyenne (ligne noire), tels que les techniciens, les professions libérales et quelques métiers ne nécessitant aucune qualification, tels que le personnel de service, les vendeurs et les ouvriers non spécialisés. La catégorie des artisans et des employés de l'artisanat affiche une tendance clairement positive proche de la moyenne de l'emploi total. Cependant, d'autres catégories telles que les législateurs, les directeurs, les opérateurs des moyens de transport et les employés de bureau, dont la tendance est positive, se trouvent en dessous de la moyenne.

Nous pouvons conclure que l'Espagne a connu deux tendances évidentes et contradictoires. D'un côté, il y a eu une tendance vers des métiers non manuels à compétences élevées; d'un autre côté, les métiers nécessitant peu de compétences atteignent un volume d'emplois important, probablement liés au développement du secteur de la construction.

Si nous comparons la structure de l'emploi en termes de professions avec les pays européens, nous constatons qu'il existe des différences considérables dans le poids des métiers non manuels et nécessitant peu de compétences. L'Espagne semble avoir un niveau plus faible de métiers non manuels.
Mais tandis que les emplois manuels continuent d'augmenter en Espagne, ils baissent dans l'Union européenne. Le niveau en Espagne reste cependant toujours inférieur aux niveaux des techniciens et des employés de bureau, et supérieurs pour les ouvriers non spécialisés. En Europe, la croissance des métiers non manuels est, dans l'ensemble, plus faible qu'en Espagne, mais la part des métiers manuels a diminué. Certains auteurs (FINA et al., 2000 et GARCÍA, 2001) ont conclu qu'une part importante des différences entre la structure de l'emploi espagnole et européenne est due aux différences observées dans la structure sectorielle plus qu'à une répartition différente des métiers au sein des secteurs. Ces différences sont particulièrement dues aux changements de la structure des métiers dans les secteurs.

\section{La flexibilisation du marché du travail, les contrats à caractère temporaire et à temps partiel}

L'histoire récente de la structure du marché du travail espagnol peut s'expliquer par une flexibilisation progressive depuis trois décennies. Depuis 1995, la croissance annuelle moyenne supérieure à $3 \%$ et la création de plus de 6 millions d'emplois ont permis d'embaucher plus de $50 \%$ de la population et de faire baisser le taux de chômage sous la barre des $9 \%$. Ce résultat a pu être obtenu grâce 
Graphique 5 : Évolution des taux d'emplois temporaires et des temps partiels en Espagne (1995-2006)

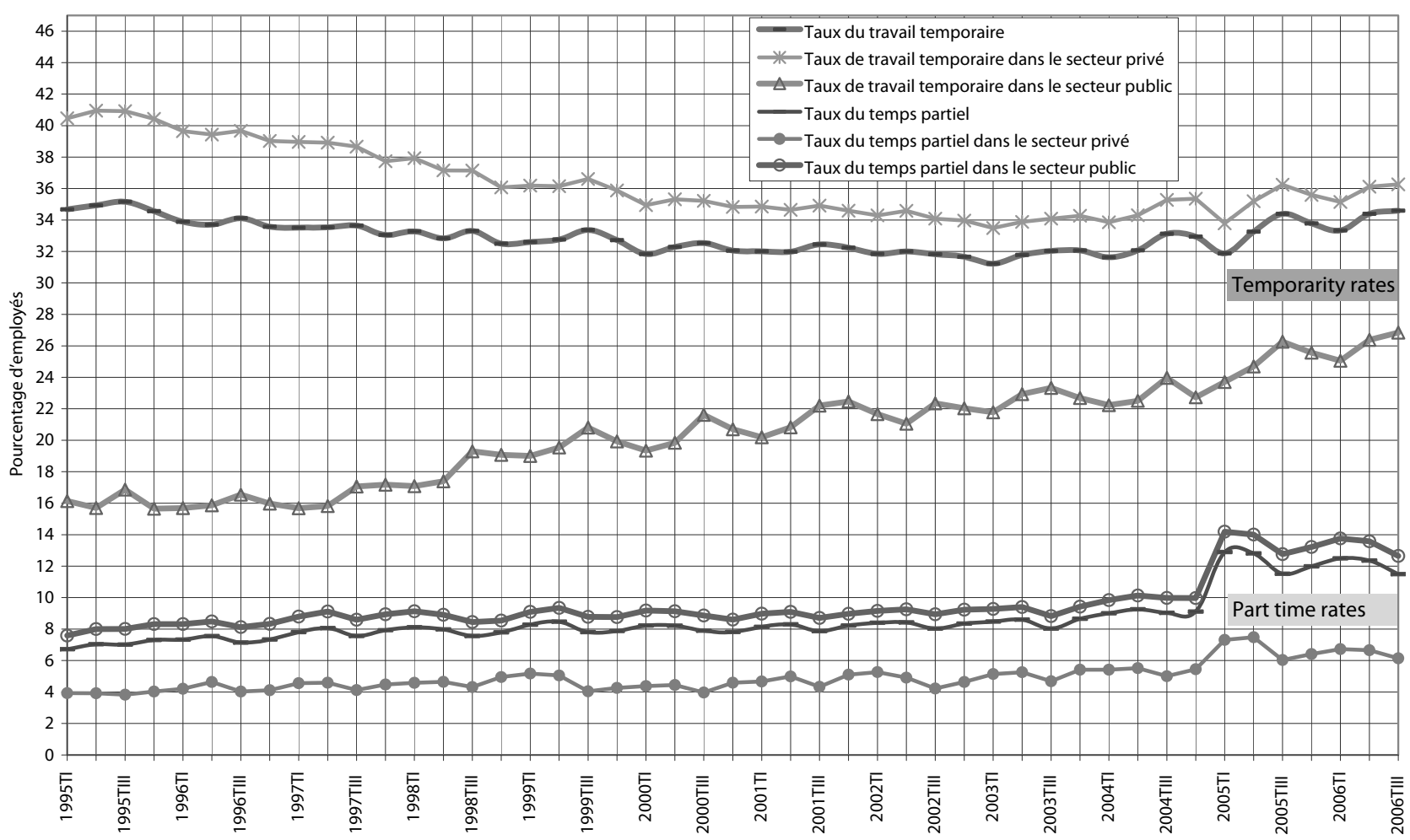

Source: Institut national de la statistique espagnol (INE), enquête sur la population active.

au recours aux diverses formes de travail temporaire (englobant diverses sortes de contrats à durée limitée ou d'intérim(6)) et, de façon marginale, au temps de travail. D'après ces éléments, l'une des caractéristiques les plus importantes des modèles d'emploi en Espagne est l'augmentation considérable de la proportion de travailleurs sur contrat «temporaire». Cette augmentation est significative depuis 1984, date d'apparition des contrats «à durée déterminée sans cause» sur le marché du travail. Leur nombre a été considéré comme «excessif» par tous les acteurs politiques, économiques et sociaux, et a engendré de nombreuses réformes juridiques (en 1994, 1997, 2001, 2006) visant à réduire l'utilisation de contrats «temporaires » par les entreprises: en limitant les fondements juridiques pour un tel contrat aux tâches de nature temporaire (1994), en réduisant les coûts de licenciement pour les travailleurs en contrat à durée illimitée et en incitant à privilégier les contrats à durée indéterminée (1997), en resserrant davantage l'utilisation autorisée de contrats temporaires $(2001,2006)$ et en encourageant la transformation des contrats à durée déterminée en contrats à durée indéterminée.

L'Espagne est le pays européen à plus forte proportion de travailleurs «temporaires» comme nous le montre le tableau 8 , bien que cette différence doive être prise en compte avec une attention particulière car certains facteurs institutionnels tels que les coûts

(6) Voir encadré 2, Miguélez et Prieto dans ce numéro spécial. de licenciement et la durée concrète des contrats, sont assez différents entre les pays de l'Union européenne. La part des employés en contrat «temporaire» au sein de l'Union européenne atteint $14 \%$ en 2005. Les chiffres sont cependant complètement différents entre l'Espagne et les autres pays européens : un tiers des emplois sont des contrats à durée déterminée en Espagne.

\section{Tableau 8 : Part de l'emploi temporaire} dans différents pays européens

et dans l'Union européenne à quinze (2005)

\begin{tabular}{|l|c|c|c|}
\hline & Hommes & Femmes & $\begin{array}{c}\text { Hommes } \\
\text { et femmes }\end{array}$ \\
\hline France & 11,6 & 13,3 & 12,4 \\
\hline Allemagne & 14,0 & 13,6 & 13,8 \\
\hline Italie & 10,6 & 14,8 & 12,4 \\
\hline Espagne & 31,6 & 35,5 & 33,3 \\
\hline Royaume-Uni & 5,2 & 5,9 & 5,5 \\
\hline $\begin{array}{l}\text { Union européenne à } \\
\text { quinze }\end{array}$ & 13,4 & 14,7 & 14,0 \\
\hline
\end{tabular}

Source: statistiques de l'OCDE.

L'augmentation du taux d'emploi à caractère temporaire a été associée à la prévalence croissante de l'emploi à temps partiel, ainsi qu'à une augmentation de la part des employés ayant un contrat «temporaire». Le graphique 5 présente l'évolution des emplois temporaires et des emplois à temps partiel en même temps que les différences entre secteur privé et secteur public. Le taux de l'emploi temporaire y apparaît comme stable depuis 1995. La 
comparaison entre le secteur privé et le secteur public souligne que la baisse depuis 1995 est plus profonde dans le privé. Il est paradoxal de constater que le secteur public a développé un modèle contractuel qui a maintenu la part des employés sur contrats «temporaires » aussi élevée (7).

La flexibilité du temps de travail, et notamment l'emploi à temps partiel, ont certainement joué un rôle important dans le développement des nouvelles formes d'emplois. Cependant, même si le gouvernement et les acteurs sociaux ont encouragé les contrats à temps partiel, leur rôle a été limité en Espagne. La tendance des contrats à durée déterminée a été combinée à un faible niveau d'emplois à temps partiel mais avec une prévalence croissante de ce type d'emploi. Cependant, comme nous pouvons l'observer dans le tableau 9, ce taux reste encore très faible en Espagne (inférieur à 12\%) par rapport aux autres pays européens, et également très faible pour les hommes (inférieur à $4 \%$ ).

Tableau 9: Part des emplois à temps partiel dans l'emploi salarié dans différents pays européens et dans l'Union européenne à quinze en 2005

\begin{tabular}{|l|r|r|r|}
\hline & Hommes & Femmes & $\begin{array}{c}\text { Hommes } \\
\text { et } \\
\text { femmes }\end{array}$ \\
\hline France & 5,6 & 23,8 & 14,3 \\
\hline Allemagne & 7,0 & 39,8 & 22,3 \\
\hline Italie & 5,0 & 30,3 & 15,7 \\
\hline Espagne & 3,7 & 22,0 & 11,4 \\
\hline Royaume-Uni & 9,4 & 38,4 & 23,7 \\
\hline $\begin{array}{l}\text { Union européenne à } \\
\text { quinze }\end{array}$ & 6,8 & 32,2 & 18,5 \\
\hline
\end{tabular}

Source: Statistiques de l'OCDE.

Le graphique 5 supra montre que les emplois à temps partiel sont moins nombreux dans le secteur public que dans le secteur privé. Cependant, le temps de travail dans le secteur public est généralement inférieur à celui du secteur privé et ne nécessite donc pas d'arrangement de temps de travail pour développer de nouvelles méthodes d'organisation(8).

Dans l'ensemble, les changements réglementaires visant à contrôler l'emploi temporaire, à développer l'utilisation de temps partiels volontaires et à encourager les emplois permanents ont connu un succès assez limité. Les réformes successives (voir encadré 2) ont atténué les rigidités du marché du travail mais n'ont pas fondamentalement changé la situation difficile sur le marché du travail puisque

(7) Pour une analyse plus détaillée du rôle des contrats «temporaires» en Espagne, voir par exemple MaLo, ToHARIA (2000) et Toharia (dir.) (2005). Pour une analyse comparative poussée sur les emplois précaires en Italie et en Espagne, voir CeBrian et al. (2002).

(8) La part des emplois à temps partiel a fortement augmenté au premier trimestre 2005 , certainement en raison des changements des méthodes de l'enquête sur les forces de travail. l'évolution du taux de l'emploi temporaire affiche clairement une tendance réellement stable et qu'un tiers des salariés font toujours partie des travailleurs temporaires. Les acteurs sociaux reconnaissent que la réforme du marché du travail est essentielle pour augmenter la productivité, ce dont l'Espagne à besoin pour faire face à la concurrence des pays en voie d'adhésion à l'Union européenne où les salaires sont plus faibles. $* \quad *$

Peut-on être sûr que la croissance de la production et de l'emploi en Espagne garde le même rythme soutenu que depuis 1994 ? Selon certains auteurs, l'évolution des taux de croissance depuis 1994 n'est due qu'à une suite d'années chanceuses qui ne dureront pas très longtemps. Ces auteurs préconisent la prudence, car le taux de croissance de la production par travailleur est très faible et ne semble pas reprendre les niveaux précédents.

La récente évolution des taux de croissance de la production et de l'emploi a sans aucun doute été très positive pour l'économie espagnole, mais elle souffre cependant de certains problèmes qui pourraient affecter la croissance future. Les niveaux de productivité sont très faibles et rien ne laisse supposer une évolution positive. Plusieurs facteurs sont en jeu ici. Premièrement, la forte croissance économique est basée sur la consommation interne et sur le secteur de la construction, mais ces deux facteurs ne sont pas les meilleurs piliers sur lesquels construire un avenir stable de croissance réelle et de bien-être avancé. Deuxièmement, les coûts unitaires du travail ont diminué, en raison d'une restriction importante des salaires et d'une faible croissance de la productivité, qui a défavorisé la part des salaires au profit des revenus du capital. Troisièmement, bien que les emplois non manuels soient en augmentation, les nouveaux emplois peu qualifiés représentent une part importante. Le modèle espagnol ne converge donc pas vers celui des pays européens les plus développés. Cet autre facteur est lié de manière négative à l'évolution positive de la productivité dans un futur proche. Enfin, le niveau très élevé des contrats à durée limitée ne semble pas favoriser de meilleurs résultats en termes de productivité.

Dans l'ensemble, il est difficile de conclure que l'économie espagnole soit passée à un stade stable de croissance économique; au contraire, il semble plus juste de penser que les facteurs de déséquilibre entraveront l'amélioration future et que l'économie ne sera pas en mesure d'avancer au même taux de croissance de l'emploi et de la production que ces dernières années. 


\section{Encadré 2}

\section{Réformes du marché du travail espagnol de 1984 à 2006}

L'histoire du marché du travail espagnol montre des contrastes importants entre l'Espagne et l'Union européenne, généralement dus à l'utilisation particulière en Espagne de "contrats temporaires sans cause", depuis la première réforme de la charte des travailleurs en 1984.

À cette occasion, des contrats temporaires spéciaux, visant à encourager l'emploi, sont apparus sans qu'une cause particulière soit requise. Ce qui signifiait que tous les chômeurs pouvaient être embauchés sur un contrat temporaire, que l'emploi soit lié ou non à une activité temporaire de l'entreprise. Ce type de contrat jouait un rôle important comme outil de flexibilité, même s'il permettait d'obtenir une flexibilité à la marge (MALo, TohaRIA, 2000) car il affectait uniquement les travailleurs embauchés sur nouveaux contrats, qui ne donnaient pas lieu à des indemnités de licenciement.

Cependant, du fait de leur augmentation en nombre et des coûts sociaux qui s'en suivaient, certaines réformes virent le jour dans le but de les limiter. L'objectif principal était de restaurer un principe de causalité. De nouvelles méthodes furent également mises en place pour diminuer la différence entre les coûts de licenciement. Les changements les plus importants introduits par les réformes eurent lieu en 1992, 1994, 1997, 2001 et 2006.

En 1992, la durée minimum des contrats «temporaires» fut allongée de six à douze mois. Par ailleurs, on incita financièrement les employeurs à préférer les contrats à durée indéterminée dans l'embauche des chômeurs de longue durée, des jeunes de moins de 29 ans et des personnes de plus de 45 ans, ainsi que certains groupes de femmes.

Ces changements ne furent pas suffisants. En 1993, une nouvelle réforme vit le jour et entra en vigueur en 1994. II s'agissait dans ce cas de retrouver un principe de causalité. II ne devenait possible d'utiliser des contrats temporaires sans cause que dans certains cas. Les conventions collectives ont joué un rôle important dans cette nouvelle période et les agences d'intérim furent autorisées.

Malgré l'ampleur des changements réglementaires introduits par la réforme de 1994, les résultats attendus ne furent pas obtenus en termes de nombre de contrats temporaires. Un accord entre les partenaires sociaux fut donc signé en 1997: l'Acuerdo Interconfederal de Estabilidad en el Empleo. Cet accord avait pour but principal d'améliorer le marché du travail et d'encourager l'emploi permanent pour combattre l'emploi temporaire et la rotation du personnel. Les contrats temporaires sans cause furent définitivement interdits et de nouvelles formes de contrats à durée indéterminée avec des coûts de licenciement plus faibles furent mises en place.

Au début, ces nouveaux contrats devaient être utilisés jusqu'en mai 2001 et étaient autorisés uniquement pour les jeunes de 18 à 29 ans, les chômeurs de plus de 45 ans, les chômeurs ayant recouru pendant au moins douze mois aux services publics de l'emploi, les travailleurs handicapés et certains travailleurs temporaires.

En 2001, une nouvelle réforme vit le jour, soutenue uniquement par le Gouvernement. Les contrats à durée indéterminée aux coûts réduits introduits en 1997 furent étendus dans le temps, ainsi que dans le nombre des catégories de travailleurs concernés: jeunes de moins de 30 ans, chômeurs ayant six mois de recherche d'emploi dans les services publics de l'emploi, femmes des secteurs à faible participation féminine, et tous les contrats «temporaires». Une indemnité de licenciement pour les contrats temporaires équivalent à huit jours de salaires par année travaillée fut également instaurée.

En 2002, les coûts salariaux furent réduits par la suppression des arriérés dus en cas de poursuites pour licenciement, Salarios de Tramitación dans le cas de licenciement abusif, si le travailleur reprenait ses fonctions dans l'entreprise.

Enfin, en 2006, une nouvelle réforme fut instaurée avec pour même objectif de réduire et de contrôler les contrats à durée déterminée. Les enchaînements de contrats «temporaires " entre une même entreprise et un même employé furent interdits après une durée totale maximale de deux ans sur une période de trente mois. Un quota fixe de pourcentage du salaire fut également instauré comme bonus pour encourager les entreprises à utiliser des contrats à durée indéterminée, notamment pour les emplois à bas salaire, pour les jeunes travailleurs, les travailleurs handicapés et les personnes en contrat de formation. La durée du bonus fut également étendue à quatre ans. 


\section{Bibliographie}

Cebrián I., Moreno G., Samek M., Semenza R., Toharia L. (2002), "Nonstandard work in Italy and Spain", in Nonstandard work arrangements in Japan, Europe and the United States, eds. Houseman, S. and Osawa, M. Kalamazoo (Mich.): W.E. Upjohn Institute for Employment Research.

COMISIÓN DE EXPERTOS PARA El DiÁLOGO SOCIAL (2005), Más y mejor empleo en un nuevo escenario socioeconómico. Por una flexibilidad y seguridad laborales efectivas, Madrid, Ministerio de Trabajo y Seguridad Social.

Cuadrado J.R., Iglesias C., Ortiz A., Guardia J. (1999), El sector servicios y el empleo en España. Evolución reciente y perspectivas de futuro, Madrid, Fundación BBV.

Fina Ll. (2001), El reto del empleo, McGraw-Hill.

Fina Ll., ToHARIA L. (2001), El empleo en España: situación y perspectivas, Madrid, Ministerio de Trabajo y Seguridad Social.

Fina Ll., Toharia L., García Serrano C., Mañé F. (2000), "Cambio ocupacional y necesidades educativas de la economía española", in Sáez F. (coord.), Formación y Empleo, Colección Economía Española, vol. XV, Fundación Argentaria-Visor, pp. 47-154.

García Serrano C. (2001), "Cambio sectorial y cambio ocupacional en España y en Europa”, in Fina and Toharia (2001) pp. 161-185.
Jimeno J.F., Moral E., SaIz L. (2006), Structural Breaks in Labor Produtivity growth: the United States and the European Union, Documentos de Trabajo $n^{\circ} 0625$, Banco de España.

Malo M.A., Toharia L. (2000), "The Spanish Experiment: the Pros and Cons of the Flexibility at the Margin", in Esping-Andersen G. Regini M., Why De-regulate Labour Markets ?, Oxford University Press, pp.307-335.

PÉrez InFAnTe J.I. (2006), Las Estadísticas del Mercado de Trabajo en España, Ministerio de Trabajo y Asuntos Sociales, Centro de Publicaciones.

SAnchez R., Toharia L. (2000), “Temporary workers and productivity: tha case of Spain", in Applied Economics, $\mathrm{n}^{\circ} 32$, pp. 583-591.

Toharia L. (2002), "Employment Patterns in Spain between 1970 and 2001 - Past Developments and Midterm Prospects», International Journal of Political Economy, Vol. 30, no 2, pp. 82-98 (published in 2002).

TOHARIA L. (dir.) (2004), "El mercado de trabajo en España: situación y perspectivas", in review CLM economía, $\mathrm{n}^{\mathrm{0}} 4$.

TOHARIA L. (dir.) (2005), El problema de la temporalidad en España: un diagnóstico, Madrid, Ministerio de Trabajo y Asuntos Sociales. 